\title{
DeVElopMent OF MACHINE LEARNing Models to DETERMINE HAND GESTURES USING EMG SigNALS
}

\author{
Vyacheslav V. Potekhin \& Ogul Unal \\ Peter the Great St. Petersburg Polytechnic University
}
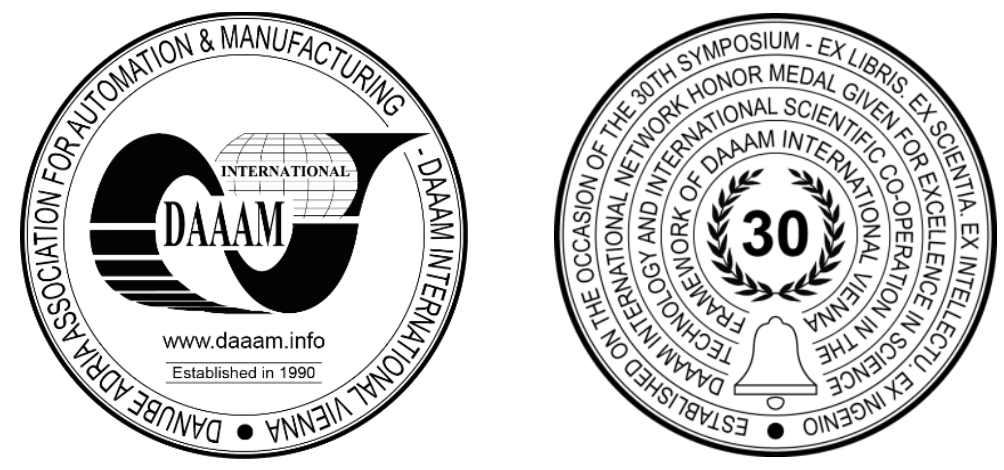

This Publication has to be referred as: Potekhin, V[yacheslav] \& Unal, O[gul] (2020). Development of Machine Learning Models to Determine Hand Gestures using EMG Signals, Proceedings of the 31st DAAAM International Symposium, pp.0261-0270, B. Katalinic (Ed.), Published by DAAAM International, ISBN 978-3-902734-29-7, ISSN 1726-9679, Vienna, Austria

DOI: $10.2507 / 31$ st.daaam.proceedings.036

\begin{abstract}
The connection between the human body and science is still growing exponentially. The human body has many mysteries. The more we learn about them, the better we improve our scientific perspectives. In this case, the analysis of EMG (Electromyographic) signals gives the possibility the use the EMG data to perform classification tasks. Machine Learning models and Neural Networks are the best tools to classify different hand gestures using the dataset. This work aims to analyse the characteristics of EMG signals and use the EMG dataset to perform different ML models. The results will be used in robotic fields and control systems as future work. In this project, the Python programming language is used. The dataset was recorded using an MYO Thalmic bracelet. The number of instances is about 40000-50000 recordings in each column (channels). There are six different hand gestures tasks recorded in the dataset that are; hand clenched in a fist, wrist extension, wrist flexion, hand radial deviations, hand ulnar deviations, hand extended palm. The study of ML models and using gestures to control robotic devices could be useful in industrial spheres. A person may use a forearm bracelet to use it in industrial operations. Another purpose of this paper is; helping people who lost their hands. With the help of robotic arm plugged-in to their fore-arm and EMG device, they might be able to perform several hand-gestures.
\end{abstract}

Keywords: Machine Learning; Random Forest; XGBoost; Electromyography; Neural Networks

\section{Introduction}

Electromyography is a unique field to understand and evaluate the electrical activity produced by skeletal muscles. Thanks to today's knowledge and technology it is not a secret how do our neurons and muscles work during our daily life. Using Electromyography, and Machine Learning, the project aims to analyse theoretical concepts of muscles and Electromyography and apply Machine Learning algorithms and make comparisons.

The medicine field has significant improvements to understand our bodies. It is not a myth or magic to control robotic equipment, systems using our hand gestures. It is discovered that the human body creates many unique signals to control its actions. These signals can be depicted as the "language" in the human body. The human brain keeps talking to our hands, arms, legs, or organs all the time. Today, with the help of many kinds of research it is likely to get this language with the help of signal processing technology and instruments and transform them into machine understandable language. 
Therefore in this project, it was possible to use Electromyography Dataset based on the human body language then classify the hand gestures using Electromyography signals. The Supervised Machine Learning algorithms were used to classify those activities and give us classification based on our gestures.

The project has significantly high accuracy results therefore, it is possible to use the Machine Learning approach in other systems. Three different algorithms were compared after analysing muscles and Electromyography. The results may be new technical solutions to perform better classifications between six different hand gestures. It is aimed to use and test the results in Cyber-Physical Systems as future work. Using Medicine researches combined with Machine Learning technologies, Human-Computer Interaction systems will be created.

\section{Literature Review}

There are more than 430 skeletal muscles paired on the right and left sides of the body. Even the most demanding movements are produced by fewer than 80 pairs. Muscles provide strength and protect the skeleton from destructive impacts, absorb jolts, allow the movement of bones with joints, and help maintain body posture against loads. These features are usually manifested by muscles that work in groups rather than a single muscle. The figure below indicates the relaxation and contraction conditions in muscles:
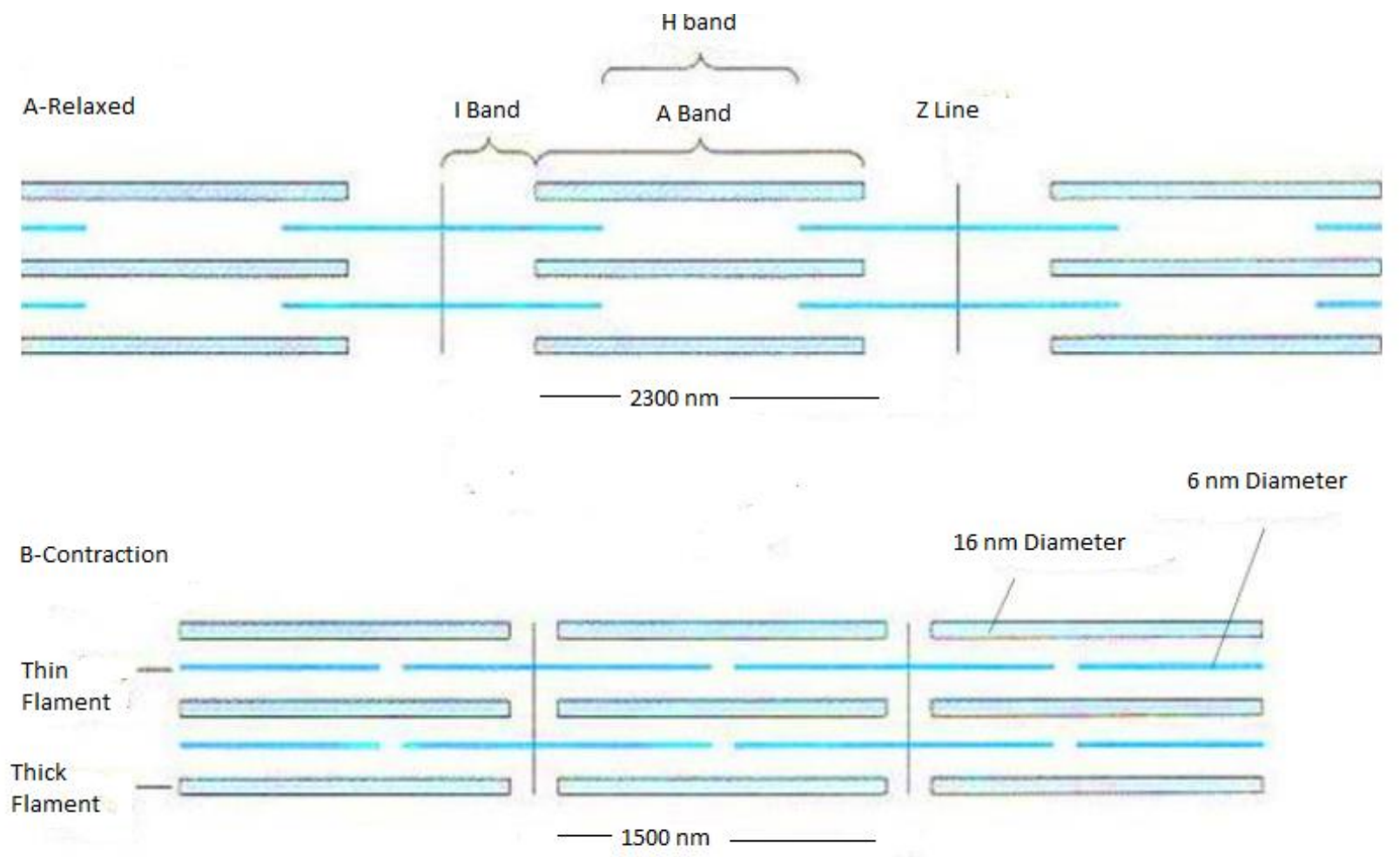

Fig. 1. Relaxation and Contraction in muscles, Figure is taken from [10]

Position of I, A, and $\mathrm{H}$ bands when relaxed. First, the fibers partially cover the ends of the thick fibers and are stuck to the Z-lines. B. Contraction condition. It is seen that the actin fibers are shifted from both sides of the myosin fibers towards each other. There is no change in the lengths of thick and thin fibers. However, the length of the sarcomere has decreased from $2300 \mathrm{~nm}$ to $1500 \mathrm{~nm}$. Two types of myofilaments are thick and thin. They are protein strands in muscle tissue that provide contraction and relaxation, which is the most important feature of muscle tissue. These muscles appear striated under the microscope because of their regular alignment in the striated (skeletal) muscle and cardiac muscle.

Andrew Huxley and his team introduced the sliding Filament theory. According to this theory: the myosin (thick) filaments of muscle fibers slide past the actin (thin) filaments during muscle contraction, while the two groups of filaments remain at a relatively constant length. The keystone of the sliding mechanism is calcium ions $(\mathrm{Ca} 2+)$ that initiate and end the contraction process. Muscle contraction begins when calcium is used as the contraction element and ends when calcium transport is stopped. The functioning of the mechanism is based on the electrical events generated by usable calcium ions in the muscle membrane (sarcolemma) [10].

Therefore, the action potential in sarcolemma generates the electrical signal necessary for the contraction to start [10]. The chemical dimension of the contraction mechanism triggered by the electrical signal is known as the arousalcontraction pair. 


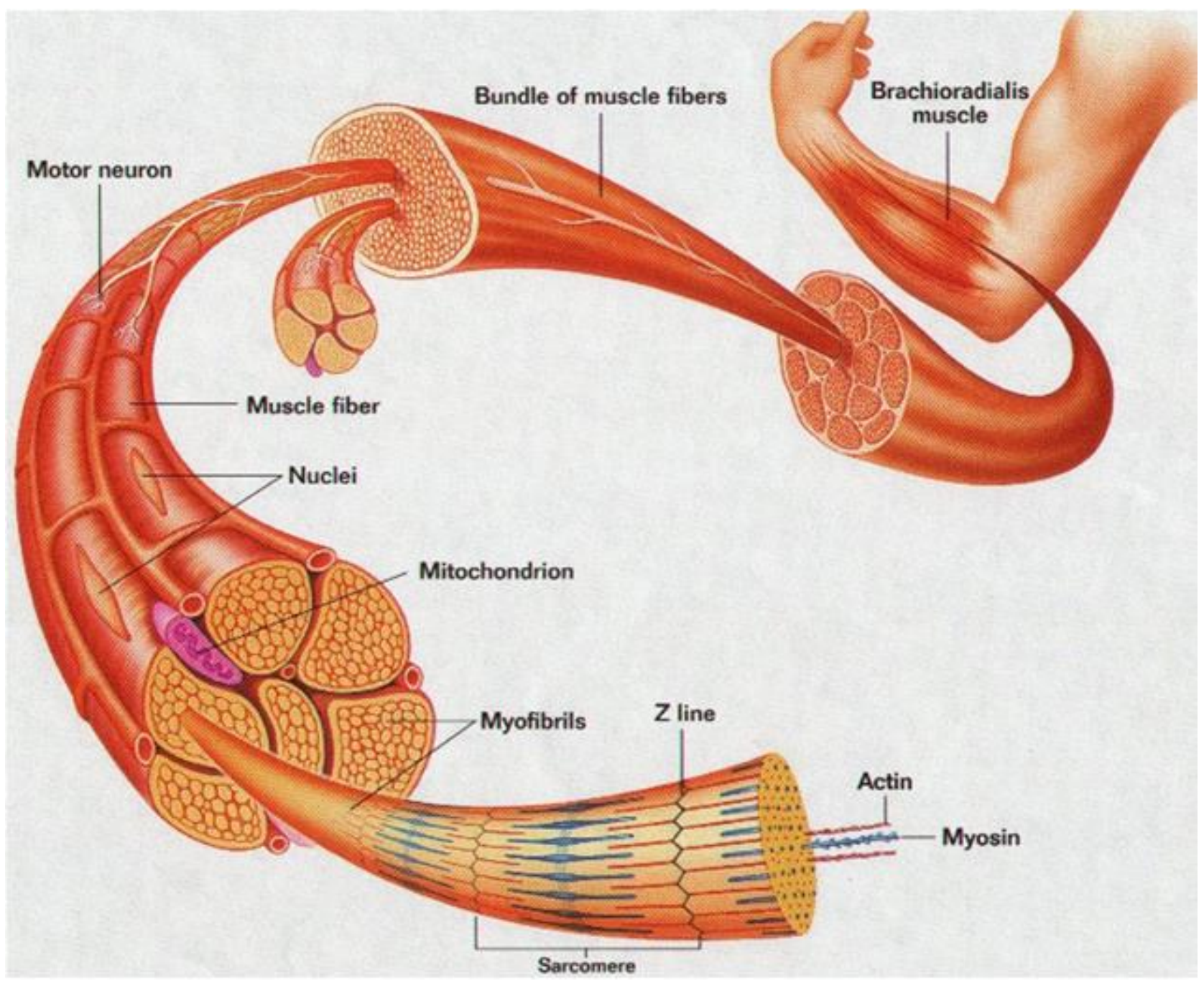

Fig. 2. The related area (forearm) and the muscle structure with neurons

Neuro physiological experiments have revealed neural correlates of many arm movement parameters, ranging from the spatial kinematics of hand path trajectories to muscle activation patterns. The levels of the motor readiness field and particularly the motor field in sensors positioned over the sensory-motor complex of the cerebral hemisphere contralateral to the hand performing the movement were entirely consistent [8]. The motor cortex controls the intentional movement. In this project, forearm gestures were performed intentionally. The signal comes from the motor cortex area in the brain [8] [13] afterward, travels through the neuron system (alpha motor neurons), and reaches to the responsible muscle connection. In neurology, the term motor neuron classifies the nerve cells (neurons) found in the central nervous system (CNS) and directly or indirectly control the muscles. Axons in the CNS transmit information to other nerve cells [12]. Motor neurons transmit signals from the spinal cord to the muscles to perform the movement. The connection between a motor neuron and muscle fiber is a special synapse called a neuromuscular junction. Depending on the adaptation stimulation, the motor neuron causes an overflow of neurotransmitters holding the synaptic receptor and the muscle fiber to react by shaking [12].

The working mechanism of the nerve-muscle junction [11]: Neural stimulation to the end of the alpha-motor nerve (1), voltage-gated calcium channels in the membrane at the tip of the nerve open, and calcium enters the cell (2). Incoming calcium, secretion containing acetylcholine (Ach) vesicles, and move their contents into the synaptic space (thin between nerve and muscle) into space) (3). Released Ach in the membrane of the opposite muscle cell It is also a cation channel, attaching to Ach receptors located enables receptors to open; this way, while $\mathrm{Na}$ enters the cell, some K cells goes out (4). Thus, the muscle cell is depolarized. Depolarization across the membrane (5), by spreading voltage-gated fast sodium channels located in adjacent areas. It causes the muscle cell to open and create an action potential (6). Formed the action potential then travels across the membrane to effect the contraction. This meanwhile, ACh-esterase, an enzyme found in the muscle cell membrane, rapidly absorbs ACh molecules. It removes its effectiveness by breaking down and reduces the efficiency of ACh molecules, mainly choline. The building blocks are taken back into the nerve cell to be used in ACh production again. (7) Figure 3 indicates the steps with numbers (). EMG measures muscle response or electrical activity. The activation starts in the brain (motor cortex) [8]. The motor cortex creates the required signals to perform muscle movements. 
The EMG tests can be used to detect muscular abnormalities as well as to create a Human-Computer-Interface. To receive the signals, at least one or more electrodes are connected around the forearm. (Surface EMG) The electrical activity picked up by the electrodes is then displayed on a device (oscilloscope). EMG measures the electrical activity of muscle during rest, slight contraction, and forceful contraction. In many cases, muscle tissue does not create electrical activity during rest. An electromyograph is made out of four components: the pre-amplifier, the transmitter-receiver couple, the hardware filters, and the analog-to-digital (A/D) [9]. The s-EMG plot is a graph of the differential voltage detected by the electrode pair plotted versus time [9]. In this project, the data is recorded in numerical format. The device that measures EMG signals is called an electromyograph, Electromyography is the electro-diagnostic technique and, the record created by electromyograph is electromyogram. Disposable electrodes generally used for s-EMG detection during motion are made out of a metallic part in $\mathrm{Ag}-\mathrm{Ag}-\mathrm{Cl}$ (electrodes) [9]. They are covered by an adhesive conductive gel (electrolyte) and surrounded by a plastic cap [9]. Machine Learning is effective when there is structured data (supervised) to perform learning. After implementing algorithms, ML can solve clustering, classification, or regression problems. In this project, it was a multi-class classification task.

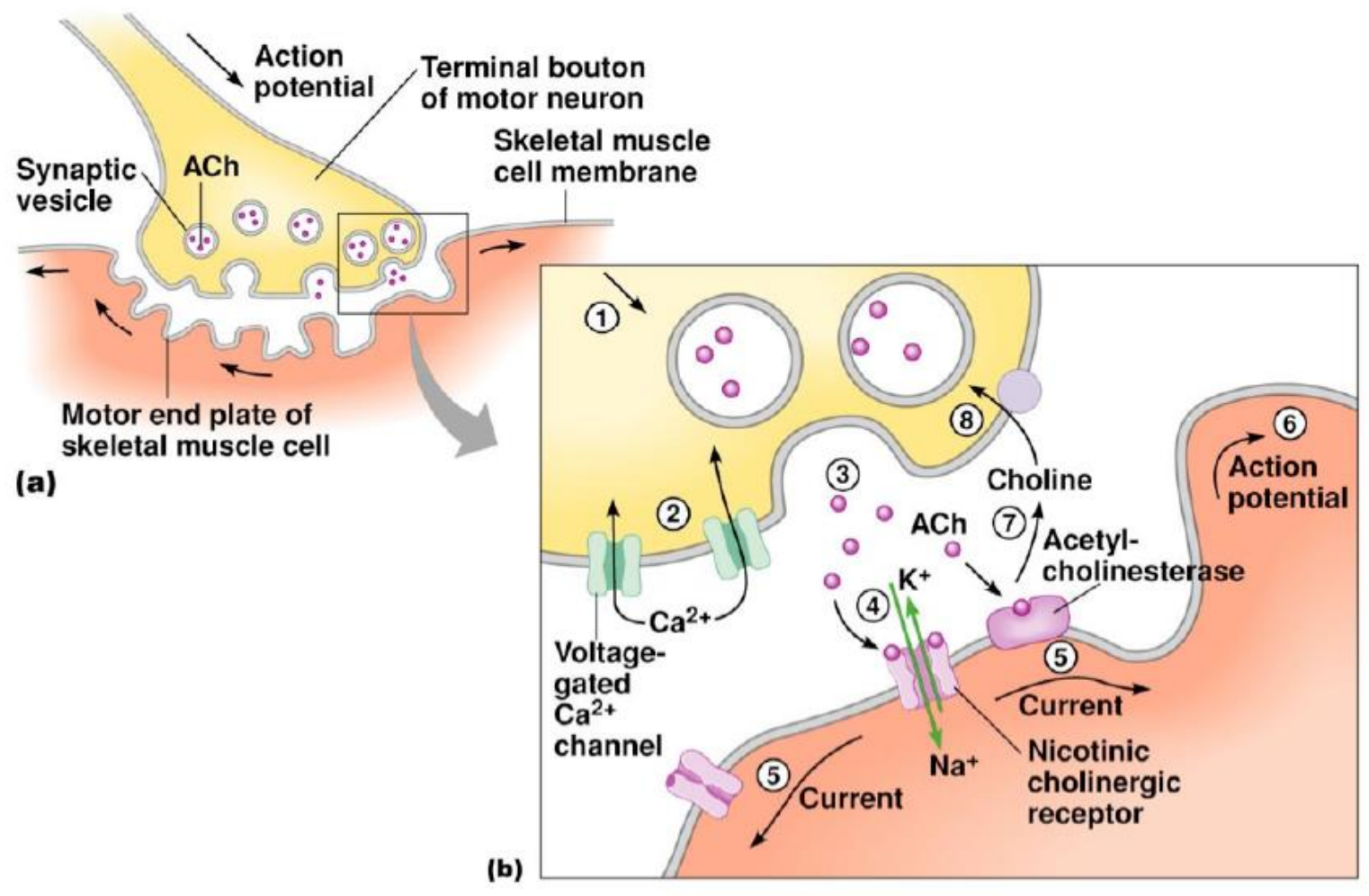

Fig. 3. The nerve-muscle junction and related areas [11]

\section{Methods \& Materials}

\subsection{EMG Device}

The MYO armband Thalmic Labs placed on the forearm was used to record the data. And this device integrates eight s-EMG sensors and an inertial measurement unit (IMU). It samples s-EMG with 8bit precision at $200 \mathrm{~Hz}$ and transmits the result to PC wirelessly using Bluetooth Low Energy (BLE) protocol [1]. To collect, process, and manage the database of received s-EMG. It has its graphical user interface (GUI) and interfaces signal processing, experiment planning, and Database management. Surface EMG (s-EMG) is the technique in which electrodes are placed on the skin overlying a muscle to record or get the electrical activity of muscles. The dataset and this article are based on s-EMG data.

The Dataset [7] used in this project has around 40000-50000 recordings for each trial; it is numerical and supervised. For recording patterns, MYO Thalmic bracelet was worn on the user's forearm, and a PC with a Bluetooth receiver. The bracelet is equipped with eight sensors equally spaced around the forearm that simultaneously acquire myographic signals [7]. Signals are transferred through a Bluetooth interface to a PC. Therefore the Dataset has its classes for seven different hand gestures represented with numbers as follows:

0 - unmarked data, 1 - hand at rest, 2 - hand clenched in a fist, 3 - wrist flexion, 4 -wrist extension, 5 - radial deviations, 6 - ulnar deviations, 7 - extended palm [7]. Therefore each number represents a specific gesture saved in the dataset. To apply machine learning algorithms, data is suitable for pre-processing, considerably clean (almost no pre-processing techniques required). More importantly, 36 different datasets were recorded from different subjects. To make comparisons and ensure the quality of machine learning techniques, at least three different datasets were used. 

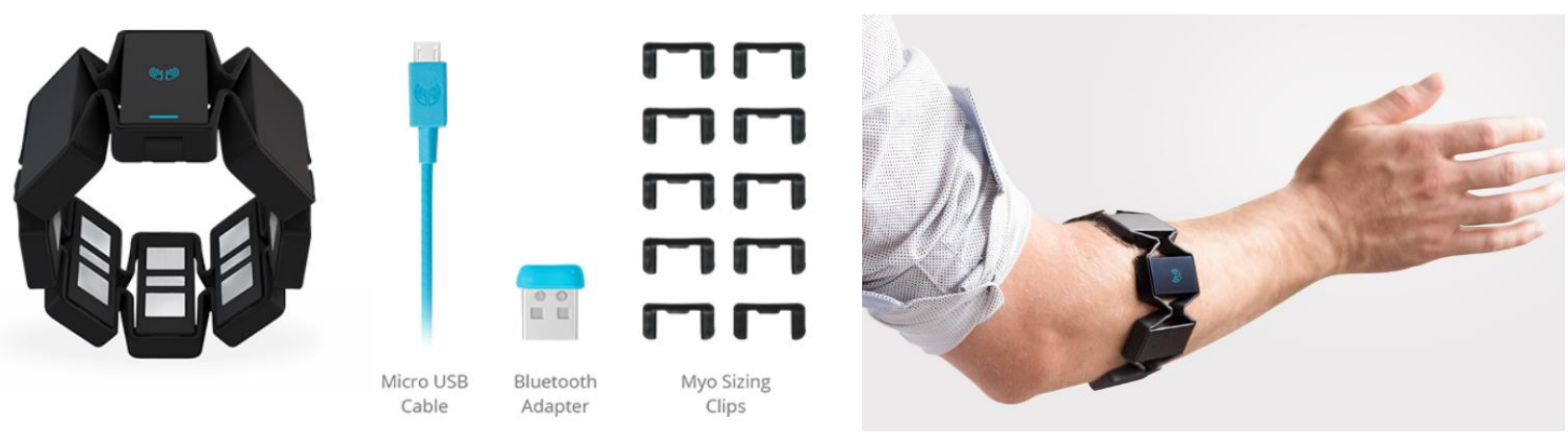

Fig. 4. The MYO armband, tools for the connection and usage placed on the forearm

\begin{tabular}{rrrrrrrrrrr} 
& time & channel1 & channel2 & channel3 & channel4 & channel5 & channel6 & channel7 & channel8 & class \\
\hline $\mathbf{0}$ & 1 & -0.00002 & -0.00001 & -0.00003 & 0.00001 & -0.00002 & -0.00001 & 0.00001 & 0.00001 & 1 \\
$\mathbf{1}$ & 5 & -0.00002 & -0.00001 & -0.00003 & 0.00001 & -0.00002 & -0.00001 & 0.00001 & 0.00001 & 1 \\
$\mathbf{2}$ & 6 & -0.00002 & -0.00001 & -0.00003 & 0.00001 & -0.00002 & -0.00001 & 0.00001 & 0.00001 & 1 \\
$\mathbf{3}$ & 7 & -0.00002 & -0.00001 & -0.00003 & 0.00001 & -0.00002 & -0.00001 & 0.00001 & 0.00001 & 1 \\
$\mathbf{4}$ & $\mathbf{8}$ & -0.00002 & -0.00001 & -0.00003 & 0.00001 & -0.00002 & -0.00001 & 0.00001 & 0.00001 & 1 \\
$\mathbf{5}$ & 9 & -0.00002 & -0.00001 & -0.00003 & 0.00001 & -0.00002 & -0.00001 & 0.00001 & 0.00001 & 1 \\
$\mathbf{6}$ & 10 & -0.00002 & -0.00001 & -0.00003 & 0.00001 & -0.00002 & -0.00001 & 0.00001 & 0.00001 & 1 \\
$\mathbf{7}$ & 11 & -0.00002 & -0.00001 & -0.00003 & 0.00001 & -0.00002 & -0.00001 & 0.00001 & 0.00001 & 1 \\
$\mathbf{8}$ & 12 & -0.00002 & -0.00001 & -0.00003 & 0.00001 & -0.00002 & -0.00001 & 0.00001 & 0.00001 & 1 \\
$\mathbf{9}$ & 13 & -0.00002 & -0.00002 & -0.00002 & -0.00003 & -0.00001 & -0.00002 & -0.00001 & -0.00001 & 1 \\
$\mathbf{1 0}$ & 14 & -0.00003 & 0.00000 & 0.00000 & -0.00002 & 0.00000 & -0.00002 & -0.00001 & -0.00001 & 1
\end{tabular}

Table 1. The dataset structure, channels, time and the target class

\subsection{Machine Learning Algorithms}

In this project, three different Machine Learning methods were used. Those are XGBoost, Random Forest, and Deep Neural Network. Ensemble Learning techniques (XGBoost and Random Forest) were used because of their high performance and high tolerance against overfitting.

As a definition; Ensemble learning combines several base models to produce one optimal predictive model. Today Industry 4.0 offers many challenging situations using big data [14]. Ensemble models in machine learning combine the decisions from multiple models to improve the overall performance. The first ML algorithm for the experiment is Random Forest. Random forests form a family of methods that consist of building an ensemble (or forest) of decision trees grown from a randomized variant of the tree induction algorithm (as described in Chapter 3). Decision trees are indeed ideal candidates for ensemble methods since they usually have low bias and high variance, making them very likely to benefit from the averaging process [2]. Random forest involves constructing a large number of decision trees from bootstrap samples from the training dataset [3]. After a large number of trees are created, they vote for the most popular result (class). The procedure is called as Random Forests.

The other chosen algorithm was the XGBoost algorithm that is another model in the field of Ensemble Learning. XGBoost is based on Decision Trees, it is an ensemble Machine Learning algorithm that uses a gradient boosting technique. The algorithm is chosen according to its original research paper [4] and results on tabular data. The s-EMG dataset is also a tabular data which means, XGBoost is a valid variation as an algorithm. XGBoost is a special implementation of the Gradient Boosting, therefore the original name is Extreme Gradient Boosting.

XGBoost applies the principle of boosting weak learners using gradient descent architecture. In this case, the Random forest is a bagging technique and XGBoost is a boosting technique. Therefore two different ensemble learning techniques were applied. The random forest takes votes of the similar or same implementations XGBoost on the other hand, improves the weights of the model. Each model, in this case, is influenced by the previous implementation. 


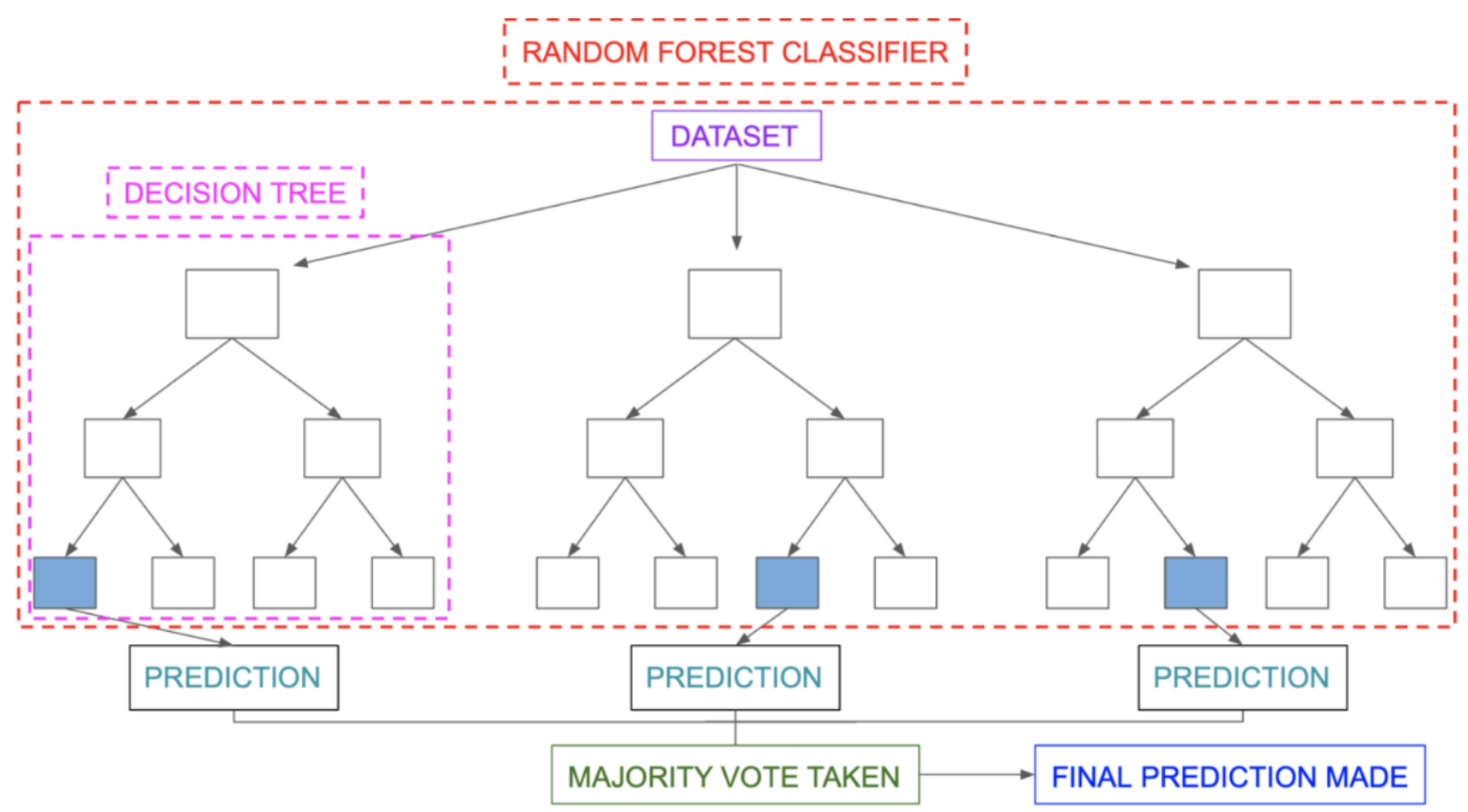

Fig. 5. Tree boosting (Random Forest) is a highly effective and widely used in machine learning

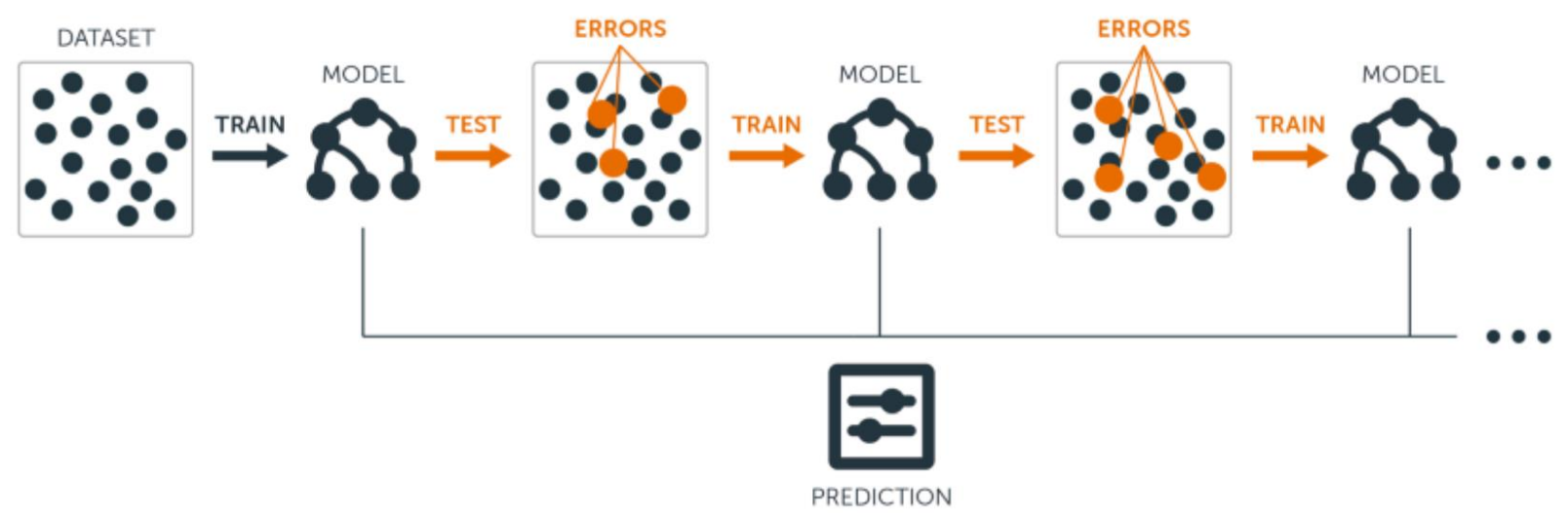

Fig. 6. The visualization of XGBoost implementation

The third approach to the problem was the usage of a Deep Neural Network. Neural Networks may give acceptable results especially when the Data-set is big enough. Before a neural network can be used for the required problem or task, it must be trained. The learning is based on given rules and data. After 500 epochs of the training, the neural network weights the connections of the neurons afterward, it develops patterns, relations which also means intelligence. The learning rules depends on how the learning material changes the neural network iteratively. Therefore the Neural Network learns how to connect the weights and relations according to its neurons properly.

The RELU (Rectified Linear Units) activation function is used in input and hidden layers. The advantages of using RELU are: it converges faster than traditional activation functions (tanh, sigmoid), and more particularly; RELU is nonsaturation of its gradient, which means it accelerates the convergence of stochastic gradient descent [6]. Therefore RELU is used as the main activation function of neurons. The Softmax gives at least a minimal amount of probability to all elements in the output vector [5]. The output values are between zero to one. Because of being able to generate any value between zero and one, Softmax is a perfect activation function when it comes to multi-class classification. Therefore in the output layer, the Softmax function is used.

The shape of the neural network is related to the number of features, in this case; the dataset has nine features for the model evaluation and training, thus the Neural network starts with nine input neurons, two hidden layers with 18 hiddenlayer units(nodes), and nine output units. Below in figure, the generated Neural Network summary is shown (python programming). 


\begin{tabular}{|c|c|c|c|c|c|}
\hline \multirow{2}{*}{ dense_7: Dense } & input: & (None, 9) & \multirow{3}{*}{$\begin{array}{l}\text { Layer (type) } \\
=:=z=-z=z= \\
\text { dense_3 (Dens }\end{array}$} & output shape & \multirow{2}{*}{ Param \# } \\
\hline & \multicolumn{2}{|l|}{ output: } & & 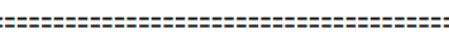 & \\
\hline & & & & $($ None, 9) & 90 \\
\hline \multicolumn{3}{|c|}{$\nabla$} & \multirow{2}{*}{ dense_ 4 (Dens } & \multirow{2}{*}{ (None, 18) } & \multirow{2}{*}{180} \\
\hline \multirow[t]{2}{*}{ dense_8: Dense } & mpal. & $(1 \times 010,2)$ & & & \\
\hline & output: & (None, 18$)$ & dense 5 (Dense & (None, 18) & 342 \\
\hline \multicolumn{3}{|c|}{$\downarrow$} & \multirow{6}{*}{$\begin{array}{l}\text { dense_ } 6 \text { (Dens } \\
\text { =-z=-z=-:=-=- } \\
\text { Total params: } \\
\text { Trainable par } \\
\text { Non-trainable }\end{array}$} & \multirow{2}{*}{ (None, 9) } & \multirow{2}{*}{171} \\
\hline \multirow{2}{*}{ dense_9: Dense } & input: & (None, 18) & & & \\
\hline & output: & (None, 18) & & \multicolumn{2}{|c|}{ 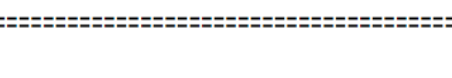 } \\
\hline \multicolumn{3}{|c|}{$\nabla$} & & & \\
\hline \multirow{2}{*}{ dense_10: Dense } & input: & (None, 18) & & & \\
\hline & output: & (None, 9) & & & \\
\hline
\end{tabular}

Fig. 7. The structure of the Neural Network of the project

\section{Results}

The muscle structure and principles of contraction and relaxation were analysed. According to this information, The Sliding Filament Theory was described. The movement of thick and thin filaments was analysed. Calcium ions (Ca2+) play a significant role during the contraction process. As soon as calcium transport stops, the contraction also stops. As a result, electrical events are generated by calcium ions in the muscle membrane.

The signals from the brain (motor cortex area) control the specific hand movements. The alpha motor neurons are transmitting the signal through neurons and reaching to the connected muscle. The nerve cells were placed on the Central Nervous System. Afterward, the working mechanism of the nerve-muscle junction was shown and described step by step.

The principles of s-EMG were described from the beginning (motor cortex) to the required muscle. The components were shown and analysed. The terminological definitions were explained.

The principles of AI were studied. The possibilities of AI were analysed [15]. The Machine Learning models were used to get results. The six different hand gestures tasks recorded in the dataset that are; hand clenched in a fist (1), wrist extension (2), wrist flexion (3), hand radial deviations (4), hand ulnar deviations (5), hand extended palm (6). The hand gestures are shown in Fig. 8.

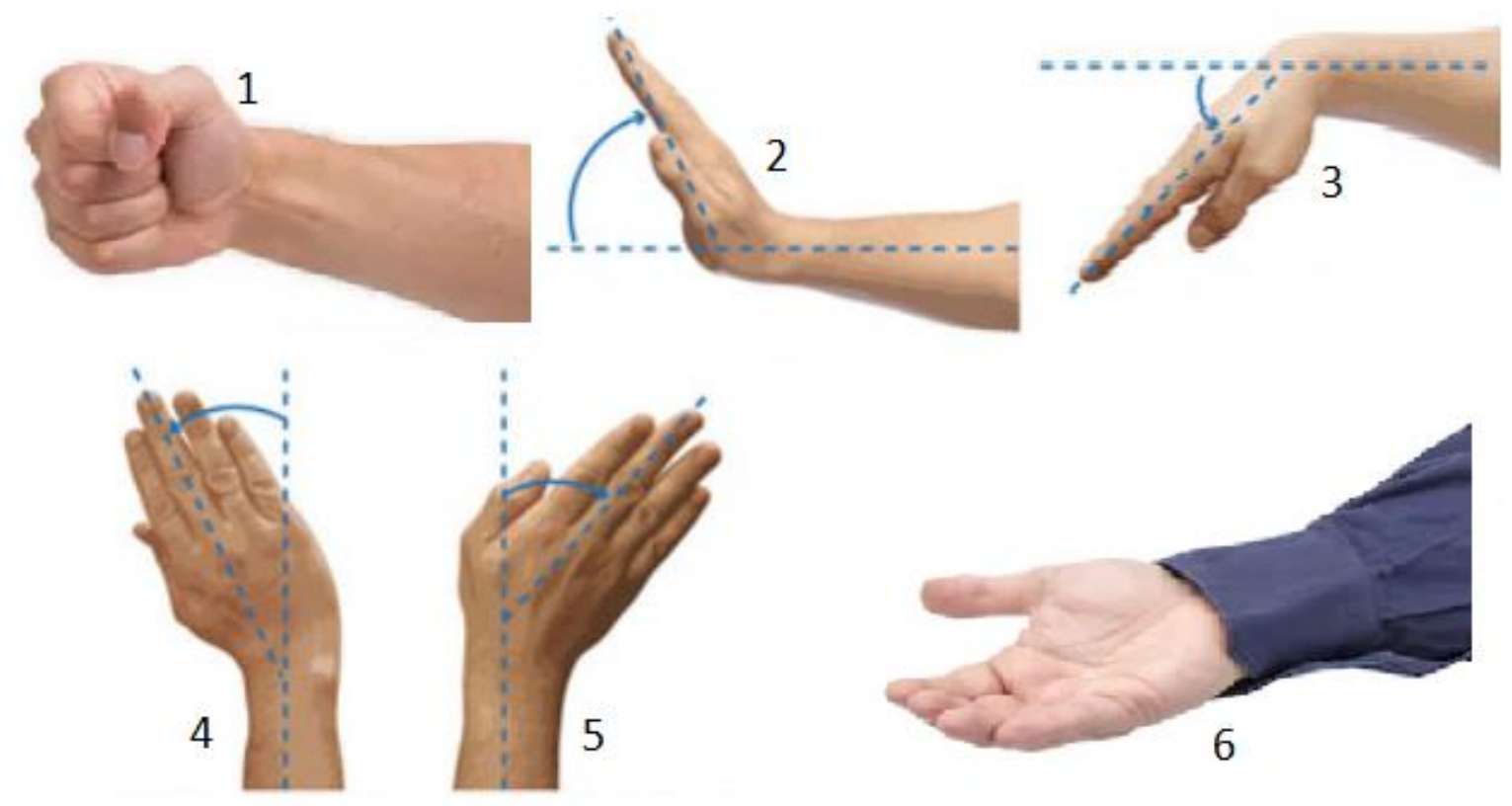

Fig. 8. The hand-gestures in the dataset 
In the dataset, the gestures are represented by the same numbers as well. The only difference is the 0 in the target column indicates the unmarked data. There are three different ML models were used. XGBoost and Random Forest algorithms were implemented. The algorithms were explained in the Methods and Materials section. The accuracy and confusion matrix is shown below for both algorithms, see Fig. 9.

A deep neural network was used. The structure and details of the built neural network where indicated in the methods and materials section. The neural network with 0.01 learning rate, ADAM optimizer, and sparse categorical cross-entropy loss function gives the following results with $97 \%$ accuracy on the EMG dataset for multi-class classification. The dataset was split into the test and training set and, the percentage was $80 \%$ training set and $20 \%$ test set.

\begin{tabular}{|c|c|c|c|c|c|c|c|}
\hline \multirow{2}{*}{$\begin{array}{r}{[[8267} \\
{\left[\begin{array}{r}8267 \\
0\end{array}\right.}\end{array}$} & 0 & 0 & 0 & 0 & 0 & 0] & \\
\hline & 845 & 0 & 0 & 0 & 0 & 0] & \\
\hline 0 & 0 & 660 & 0 & 0 & 0 & $0]$ & \\
\hline 0 & 0 & 0 & 645 & 0 & 0 & 0] & \\
\hline 1 & 0 & 0 & 0 & 641 & 0 & 0] & \\
\hline 0 & 0 & 0 & 0 & 0 & 633 & $0]$ & \\
\hline \multirow[t]{8}{*}{0} & 0 & $\begin{array}{c}0 \\
\text { pre }\end{array}$ & $\begin{array}{c}0 \\
\text { cision }\end{array}$ & 0 & $\begin{array}{c}0 \\
\text { recall }\end{array}$ & 637]] & support \\
\hline & 0 & & 1.00 & & 1.00 & 1.00 & 8267 \\
\hline & 1 & & 1.00 & & 1.00 & 1.00 & 845 \\
\hline & 2 & & 1.00 & & 1.00 & 1.00 & 660 \\
\hline & 3 & & 1.00 & & 1.00 & 1.00 & 645 \\
\hline & 4 & & 1.00 & & 1.00 & 1.00 & 642 \\
\hline & 5 & & 1.00 & & 1.00 & 1.00 & 633 \\
\hline & 6 & & 1.00 & & 1.00 & 1.00 & 637 \\
\hline $\mathrm{acc}$ & uracy & & & & & 1.00 & 12329 \\
\hline macr & o avg & & 1.00 & & 1.00 & 1.00 & 12329 \\
\hline eighte & d avg & & 1.00 & & 1.00 & 1.00 & 12329 \\
\hline
\end{tabular}

\begin{tabular}{|c|c|c|c|c|c|c|}
\hline$[8260$ & 0 & 2 & 0 & 1 & 2 & 2] \\
\hline$[0$ & 845 & 0 & 0 & $\theta$ & 0 & 0] \\
\hline 9 & 0 & 651 & 0 & 0 & 0 & 0] \\
\hline 4 & 0 & 0 & 641 & 0 & 0 & 0] \\
\hline 15 & 0 & 0 & 0 & 627 & 0 & 0] \\
\hline 4 & 0 & 0 & 0 & 0 & 629 & 0] \\
\hline 7 & 0 & 0 & 0 & 0 & 0 & 630] \\
\hline
\end{tabular}

XGBBOOSt: $99.99188904209588 \%$

Fig. 9. The results of the algorithms for testing

$\begin{array}{rrrrr}0 & 1.00 & 1.00 & 1.00 & 8267 \\ 1 & 1.00 & 1.00 & 1.00 & 845 \\ 2 & 1.00 & 0.99 & 0.99 & 660 \\ 3 & 1.00 & 0.99 & 1.00 & 645 \\ 4 & 1.00 & 0.98 & 0.99 & 642 \\ 5 & 1.00 & 0.99 & 1.00 & 633 \\ 6 & 1.00 & 0.99 & 0.99 & 637 \\ & & & & \\ \text { accuracy } & & & 1.00 & 12329 \\ \text { macro avg } & 1.00 & 0.99 & 0.99 & 12329 \\ \text { weighted avg } & 1.00 & 1.00 & 1.00 & 12329\end{array}$

Random Forest: $99.6268959364101 \%$

support

Epoch 495/500

49841/49841 [e===========================] - 1s 14us/sample - loss: 0.0924 - acc: 0.9628 - val_loss: 0.1050 - val_acc: 0.95

10

Epoch $496 / 500$

49841/49841 [ $===========================$ ] - 1s 15us/sample - loss: 0.0746 - acc: 0.9702 - val_loss: 0.1064 - val_acc: 0.95 41

Epoch $497 / 500$

49841/49841 [=============================] - 1s 15us/sample - loss: 0.0717 - acc: 0.9714 - val_loss: 0.0621 - val_acc: 0.97 38

Epoch $498 / 500$

49841/49841 [============================] - 1s 17us/sample - loss: 0.0833 - acc: 0.9659 - val_loss: 0.0535 - val_acc: 0.97 93

Epoch $499 / 500$

49841/49841 [e===========================] - 1s 16us/sample - loss: 0.0838 - acc: 0.9664 - val_loss: 0.0745 - val_acc: 0.96 65

Epoch 500/500

49841/49841 [ $============================$ ] - 1s 14us/sample - loss: 0.0779 - acc: 0.9678 - val_loss:0.0553 - val_acc: 0.97
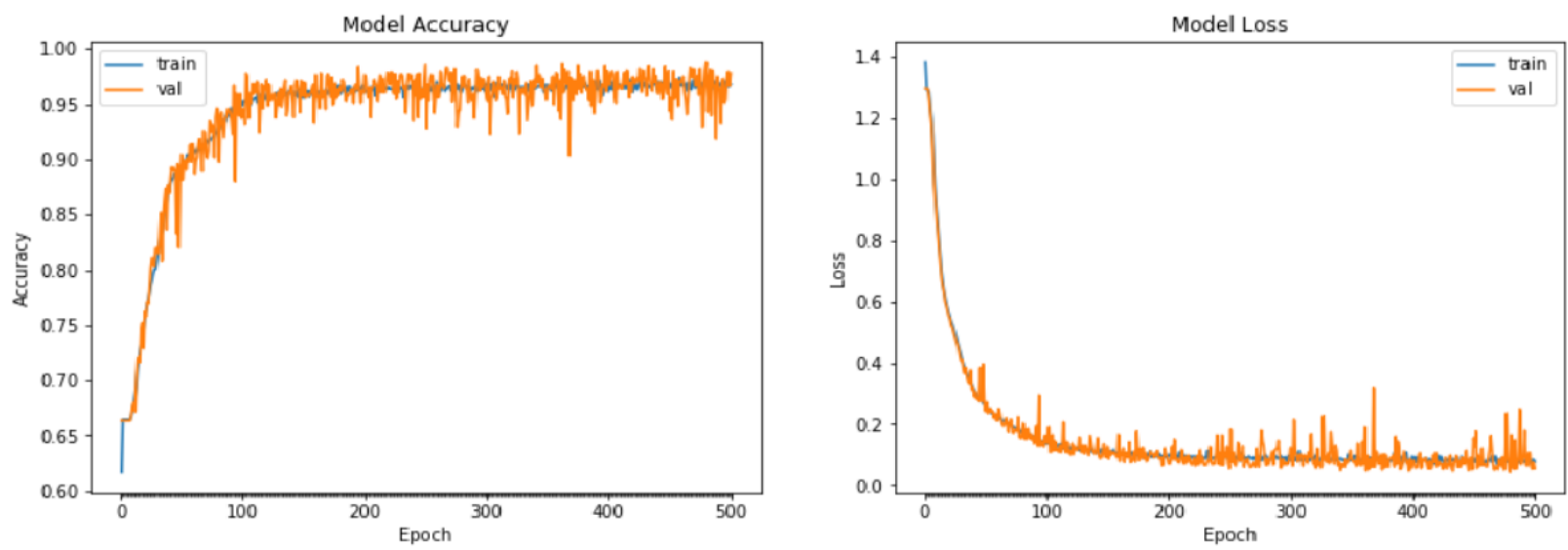

Fig. 10. The final five epochs of the training and the results (accuracy and loss) of the DNN

\section{Discussion}

The muscular activity in the human brain is analyse. Respectively, the Motor cortex sends the signal via alpha motor neurons to the required forearm muscle. During muscle activities, individual signals are created, and using the s-EMG device, signals are collected and used for ML algorithms as a dataset. 
All three algorithms in the experiment have successfully classified the dataset. The EMG data was relatively easy to perform classification. EMG is the strongest of the signals produced by the human body. The amplitude of the sign can be up to 1-2 volts in some individuals. Therefore the dataset created by the signals was significant enough to have high accuracies. All three accuracies were over 95\%. Random forest and XGBOOST gave over 99\% accuracy. The result of the Neural Network, on the other hand, was 97\% accuracy. But according to the NN results, there might be better regularization techniques to use in the future. The model accuracy and the model loss graph shows the instability during training (during 500 epochs). The dataset is big enough to experiment with more different algorithms such as Support Vector Machine, different Neural Network architectures, and a Recurrent Neural Network to analyse and compare the results.

After successfully classifying six different hand gestures, and having high accuracies from particular models, each model might be used in robotic systems to classify the incoming real-time EMG signals from the forearm. The Signals could be interpreted and pre-processed. Afterward, using ML algorithms, robotics, industrial devices can be controlled using EMG signals and ML models. For future work, other algorithms will have experimented and the model will be tested using Cyber-Physical Systems [16].

\section{Conclusion}

In conclusion, muscles in the human body work together to perform movements or actions. Muscle movements depend on relaxation and contraction mechanism. The action command comes with the alpha motor neuron and triggers chemical activity around the nerve-muscle junction. The procedure creates the action potential to perform gestures or actions using our muscles. EMG activity can be used to determine the unique hand gestures. The muscular activity generates individual signals thus, it is possible to detect specific hand gestures. The EMG signals are clear enough to perform multi-class classification using Machine Learning algorithms. The classification accuracies are significantly high enough to use the model in real-time equipment. In the future, the Machine Learning model will be tested using Cyber-Physical and control systems. Finally, the EMG signals can be used to develop artificial intelligence to mimic hand gestures.

\section{Acknowledgements}

Firstly, I would like to thank my father Dr. Turgay Ünal for his help in EMG studies and muscular activity researches. I would like to thank Associate professor Vyacheslav Potekhin for all his help during my studies. Finally, I thank my mom Dr. Seher Gülçin Ünal for her assistance in giving all the support in my life.

The paper is published in the framework of the project Erasmus+ 573545-EPP-1-2016- 1-DE-EPPKA2-CBHE-JP and describes the part of the project conducted by Peter the Great St. Petersburg Polytechnic University and also with the support of a program World Level Research Centre "Advanced Digital Technologies" in the direction of research "Promising Platform Solutions for the Integration of Industrial Technologies of Cyber-Physical and Artificial Intelligence Systems."

\section{References}

[1] Konstantin Akhmadeev, Elena Rampone, Tianyi Yu, Yannick Aoustin, Éric, Le Carpentier (2018) A real-time gesture classification using surface Emg to control a robotics hand, Enoc 2017, Hal Archives Ouvertes, Budapest, hal-01707679

[2] Gilles Louppe (2014). Understanding Random Forests from theory to practice, Ph.d. Dissertation, Department of Electrical Engineering \& Computer Science, University of Liège, Liège, Belgium

[3] Max Kuhn, Kjell Johnson (2018). Applied Predictive Modeling, Springer, ISBN 978-1-4614-6849-3, New York

[4] Tianqi Chen, Carlos Guestrin (2016). Xgboost A Scalable Tree Boosting System, the 22nd Acm Sigkdd, International Conference, pp.1-13., ISSN 978-1-4503-4232-2, doi:10.1145/2939672.2939785

[5] Michael Nielsen (2019) Neural Networks and Deep Learning, Determination Press, Unpublished

[6] Alex Krizhevsky, Ilya Sutskever, Geoffrey E. Hinton (2012) ImageNet Classification with Deep Convolutional Neural Networks, Advances in neural information processing systems, pp. 1-9., ISSN 1049-5258, doi: $10.1145 / 3065386$

[7] Lobov S., Krilova N., Kastalskiy I., Kazantsev V., Makarov V.A (2018) Latent Factors Limiting the Performance of sEmg-Interfaces, Sensors (Sensors-Basel), Basel, pp. 1-19., ISSN 1424-8220, doi: 10.3390/s18041122

[8] Dr. Vyacheslav V. Potekhin, Muhammed E. Topcu, Ogul Unal (2018) Development of Artificial Neural Network To Determine Hand Activity Using Real Motor Activity From Electroencephalography Signals, In: Apple Space Engineering, Technologies \& Exploration, Dr. Arnold Sterenharz, Alexandra Ivanovapp, (Ed.), pp. 214-224., Ecm Space Technologies GmbH, ISBN 978-3-00-060580-2, Berlin

[9] Andrea Merlo, Isabella Campanini (2010) Technical Aspects of Surface Electromyography for Clinicians, The Open Rehabilitation Journal, Bentham Open, pp. 1-13., ISSN 1874-9437, doi:10.2174/1874943701003010098

[10] Kasım Serbest, Osman Eldogan (2014) Biomechanics and Structure of Skeletal, pp. 1-11., Academic PlatformJournal of Engineering and Science pp.1-11., ISSN 2147-4575, doi: 10.5505/apjes.2014.70299 
[11] Elaine N. Marieb, Katja N. Hoehn (2009). Anatomy \& Physiology, Pearson International Edition 3rd Edition, ISBN 0321488164, Calgary

[12] C.J. Heckman, Roger Enoka (2004) Physiology of the motor neuron and the motor unit, pp. 119-149., Handbook of Clinical Neurophysiology, Amsterdam, ISSN 1567-4231, doi: 10.1016/S1567-4231(04)04006-7

[13] Kapralov, N.V., Ekimovskii, J.V., Potekhin, V.V (2020) Eeg-Based Brain-Computer Interface for Control of Assistive Devices, pp. 536-543., Lecture Notes in Networks and Systems, doi: 10.1007/978- 3-030-34983-7 52

[14] Bajic, Bojana, Cosic Ilija, Katalinic Branko, Moraca Sloboda, Lazarevic Milovan, Rikalovic Aleksandar(2019) Edge Computing vs. Cloud Computing: Challenges and Opportunities in Industry 4.0, Proceedings of the 30th DAAAM International Symposium, ISSN 1726-9679, ISBN 978-3-902734 -22-8, B. Katalinic (Ed.), pp.0864-0871., Published by DAAAM International, Vienna, doi: 10.2507/30th.daaam.proceedings.120

[15] Tekic, Zeljko; Cosic, Ilija, Katalinic, Branko (2019). Manufacturing and the Rise of Artificial Intelligence: Innovation Challenges, Proceedings of the 30th DAAAM International Symposium, ISSN 1726-9679, ISBN 9783-902734-22-8, B. Katalinic (Ed.), pp. 0192-0196., Published by DAAAM International, Vienna, doi: 10.2507/30th.daaam.proceedings.025

[16] Katalinić, B., Kostenko, D., Onufriev, V.A., Potekhin, V.V (2020) Cyber-Physical Systems in Complex Technologies and Process Control, pp.40-54., Lecture Notes in Networks and Systems, doi: 10.1007/978-3-03034983-7_5 